\title{
Ohne Leid
}

_ Einfach einschlafen! Das wünsche ich mir, wie die meisten, wenn sie gefragt werden, wie sie sterben möchten. Schmerzfrei soll es sein und ohne vorherigen Leidensweg. Ein Wunsch, den der deutsche Lyriker Emanuel Geibel Mitte des 19. Jahrhunderts in einem Gedicht sehr anschaulich beschrieben hat: „Ich möchte sterben wie der Schwan - Der, langsam rudernd mit den Schwingen - Auf seiner blauen Wasserbahn - Die Seele löst in leisem Singen [...].“

_ Ich weiß, dass nicht jedem von uns dieser Wunsch erfüllt werden wird. Zu viele Krankheiten können unseren Lebensweg kreuzen und uns bis zum Tod begleiten. Dann aber will ich die letzten Jahre würdevoll verbringen, keine Schmerzen haben und nicht vereinsamen. Die Palliativmedizin stell sich dieser Aufgabe. Ihre Geburtsstunde war 1967, als Cicely Saunders in London das St. Christopher's Hospiz gründete. Eine Einrichtung, die erstmals ambulante Versorgungsstrukturen in Form von Hausbetreuungsdiensten als Ergänzung zu stationären Angeboten integrierte. In Deutschland dauerte es noch einige Jahre, bis 1983 die erste Station für Palliativmedizin an der Universitätsklinik Köln eröffnet wurde. Und das erste Hospiz öffnete erst 1986 in Aachen seine Tore.

_ Seit 2007 gibt es hierzulande sogar einen gesetzlichen Anspruch auf spezialisierte ambulante Palliativversorgung (SAPV). Ziel war es, bis 2010 eine flächendeckende Betreuungsstruktur zu schaffen, die jährlich 80.000 Sterbende erreicht. „Tatsächlich haben im vergangenen Jahr aber nicht einmal 4.000 Menschen SAPV-Leistungen bekommen“, schätzt die Patientenschutzorganisation Deutsche Hospiz Stiftung.

_ Eine Unterversorgung, die uns zum Handeln motivieren sollte. Dieser Zweig des Gesundheitswesens hat deutlich mehr Aufmerksamkeit verdient, und Krankenkassen und Leistungsanbieter sollten sich gleichermaßen dafür einsetzen. Schön ist es da zu sehen, dass sich der Arbeitskreis Physiotherapie in der Deutschen Gesellschaft für Palliativmedizin spürbar engagiert (www.dgpalliativmedizin.de). Zum Beispiel informiert er in einem eigenen Newsletter seit letzten September über seine Aktivitäten und Fortbildungsangebote. Letztere stellen wir Ihnen in dieser physiopraxis-Ausgabe vor (๑ S. 42). Vielleicht stößt der Beitrag ja den ein oder anderen an, sich vermehrt in diesem schwierigen, aber wichtigen Arbeitsfeld zu engagieren.

- Ihre

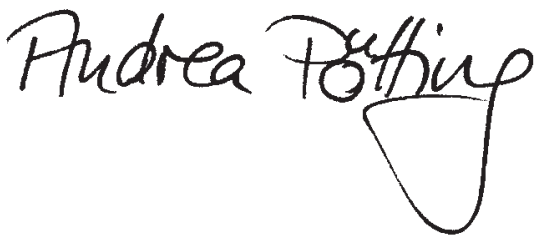

\section{PHYSIOBONUS}

physiobonus heißen die Gewinnspiele in physiopraxis. Wer teilnehmen möchte, nutzt das Formular unter www.thieme.de] physioonline in der Rubrik „physioexklusiv". Und das gibt es in dieser Ausgabe zu gewinnen:

\begin{tabular}{|c|c|}
\hline $\begin{array}{l}\text { Kursplatz } \\
1 \text { Kursplatz „Therape }\end{array}$ & ches \\
\hline Coaching“" & Seite 61 \\
\hline $\begin{array}{l}\text { Bücher } \\
\text { 2-mal „Wachkoma“ }\end{array}$ & Seite 26 \\
\hline $\begin{array}{l}\text { 2-mal „Der Mann, } \\
\text { der wirklich liebte“ }\end{array}$ & Seite 26 \\
\hline $\begin{array}{l}\text { 2-mal „Atem } \\
\text { und Bewegung“ }\end{array}$ & Seite 39 \\
\hline $\begin{array}{l}\text { 2-mal „Assessments } \\
\text { in der Rehabilitation } \\
\text { Bd. } 1 \text { Neurologie“ }\end{array}$ & Seite 40 \\
\hline $\begin{array}{l}\text { 3-mal „Kulturschock } \\
\text { Japan““ }\end{array}$ & Seite 45 \\
\hline
\end{tabular}

